\title{
The properties of solutions of certain type of difference equations
}

\author{
Xiaoguang Qi ${ }^{1 *}$, Jia Dou ${ }^{2}$ and Lianzhong Yang ${ }^{3}$
}

${ }^{*}$ Correspondence: xiaogqi@gmail.com

'School of Mathematics, University of Jinan, Jinan, Shandong 250022, P.R. China

Full list of author information is available at the end of the article

\begin{abstract}
In this paper, we shall utilize Nevanlinna value distribution theory to study the solvability of the difference equations of the form: $f(z)^{n}+p(z)\left(\Delta_{C} f\right)^{m}=r(z) e^{q(z)}$ and $f(z)^{n}+p(z) e^{q(z)}\left(\Delta_{c} f\right)^{m}=r(z)$, and we shall study the growth of their entire solutions. Moreover, we will give a number of examples to show that the results in this paper are the best possible in certain senses. This article extends earlier results by Liu et al. (Czechoslov. Math. J. 61:565-576, 2011; Ann. Pol. Math. 102:129-142, 2011).

MSC: Primary 39A05; secondary 30D35
\end{abstract}

Keywords: meromorphic functions; difference equation; growth; finite order

\section{Introduction and main results}

In this paper, we use the basic notions in Nevanlinna theory of meromorphic functions, as found in [1]. In addition, we use $\delta(f), \lambda(f)$, and $\lambda\left(\frac{1}{f}\right)$ to denote the order, and the exponents of the convergence of zeros and poles of a meromorphic function $f(z)$, respectively. We define difference operators as $\Delta_{c} f=f(z+c)-f(z)$, where $c$ is a non-zero constant.

In 2001, Yang [2] started to study the existence and uniqueness of finite order entire solutions of the following type of non-linear differential equation:

$$
L(f)-p(z) f(z)^{n}=h(z)
$$

where $L(f)$ is a linear differential polynomial in $f$ with polynomial coefficients, $p(z)$ is a non-vanishing polynomial, $h(z)$ is an entire function, and $n$ is an integer such that $n \geq 4$. Subsequently, several papers have appeared in which the solutions of (1) are studied. The reader is invited to see [3-5].

Recently, Yang and Laine [6] considered the existence of the non-linear differentialdifference equation of the form

$$
f(z)^{n}+L(z, f)=h(z)
$$

where $L(z, f)$ is a finite sum of product of $f$, derivatives of $f$, and their shifts. They obtained the following result.

Theorem A Let $n \geq 4$ be an integer, $L(z, f)$ be a linear differential-difference polynomial of $f$, with small meromorphic coefficients, and $h(z)$ be a meromorphic function of finite order.

\section{照 Springer}

@2014 Qi et al.; licensee Springer. This is an Open Access article distributed under the terms of the Creative Commons Attribution License (http://creativecommons.org/licenses/by/2.0), which permits unrestricted use, distribution, and reproduction in any medium, provided the original work is properly cited. 
Then (2) possesses at most one admissible transcendental entire solution of finite order, unless $L(z, f)$ vanishes identically. If such a solution $f(z)$ exists, then $f(z)$ is of the same order as $h(z)$.

In particular, it is shown in [6] that the equation

$$
f(z)^{2}+P(z) f(z+1)=Q(z)
$$

has no transcendental entire solution of finite order, where $P(z), Q(z)$ are polynomials.

Some improvements of Theorem A can be found in [7-10] as well. Replacing $f(z+c)$ with $\Delta_{c} f$, one of the present authors considered the existence of entire solutions of

$$
f(z)^{n}+P(z)\left(\Delta_{f} f\right)^{m}=Q(z)
$$

we get the following result in [11].

Theorem B Let $P(z), Q(z)$ be polynomials, $n$ and $m$ be integers satisfying $n>m \geq 0$. Then (3) has no transcendental entire solution of finite order.

In fact, the special case of (3) with $n=m$, and $p(z)=q(z)=1$, can be viewed as the Fermat type functional equation. It is well known that (3) has no transcendental entire solutions when $n \geq 3$, which can be seen in [12].

It is natural to ask what happens if $P(z)$ or $Q(z)$ is a transcendental entire function in (3). Corresponding to this question, we will investigate the finite order entire (meromorphic) solution of

$$
f(z)^{n}+p(z)\left(\Delta_{c} f\right)^{m}=r(z) e^{q(z)} \quad \text { and } \quad f(z)^{n}+p(z) e^{q(z)}\left(\Delta_{c} f\right)^{m}=r(z) .
$$

Theorem 1.1 Consider the non-linear difference equation of the form

$$
f(z)^{n}+p(z)\left(\Delta_{c} f\right)^{m}=r(z) e^{q(z)},
$$

where $p(z) \not \equiv 0, q(z), r(z)$ are polynomials, $n$ and $m$ are positive integers. Suppose that $f(z)$ is a transcendental entire function of finite order, not of period $c$. If $n>m$, then $f(z)$ cannot be a solution of $(4)$.

Remarks (1) In case $n \leq m$, Theorem 1.1 is not true. In the special case that $m=n=1$, the function $f(z)=e^{z}$ solves

$$
f(z)+z \Delta_{\ln 2} f=(z+1) e^{z} .
$$

Moreover, the entire function $f(z)=e^{z}+z$ is a solution of the following equation:

$$
f(z)+\frac{z}{4 \pi}\left(\Delta_{2 \pi i} f\right)^{2}=e^{z} .
$$

(2) From Theorem 1.1, we get the following result: Let $f(z)$ be a transcendental entire function of finite order, then for $n>m, f(z)^{n}+p(z)\left(\Delta_{c} f\right)^{m}$ assumes zero infinitely often, where $p(z) \not \equiv 0$ is polynomial. 
In the following, we will consider the properties of the solutions of the equation

$$
f(z)^{n}+p(z) e^{q(z)}\left(\Delta_{f} f\right)^{m}=r(z)
$$

where $p(z) \not \equiv 0, q(z), r(z)$ are polynomials. In fact, (5) may have solutions. For example, the function $f(z)=z e^{z}$ is a solution of the equation $f(z)^{3}-\frac{z^{3}}{2 \pi i} e^{2 z} \Delta_{2 \pi i} f=0$. One solution of the equation $f(z)-e^{z} \frac{1}{2 \pi i} \Delta_{2 \pi i} f=z$ is the function $f(z)=e^{z}+z$. In addition, the function $f(z)=e^{z}+z$ can solve $f(z)+e^{z} \frac{1}{4 \pi}\left(\Delta_{2 \pi i} f\right)^{2}=z$ as well. Hence, here we just study the order of the growth of the solutions. We state our findings as follows.

Theorem 1.2 Let $p(z) \not \equiv 0, q(z), r(z)$ be polynomials, $n$ and $m$ be positive integers satisfying $n>m$. Let $f(z)$ be finite order entire solutions of $(5)$, then $\delta(f)=\operatorname{deg} q(z)$.

Remark We will give the following examples to show that the assumption that $n>m$ in Theorem 1.2 is sharp. Clearly, $f(z)=e^{z}$ is a solution of the equation $f(z)-\frac{1}{2} \Delta_{\ln 2} f=0$. The function $f(z)=4 e^{8 \pi i z}-e^{4 \pi i z}+z$ solves $f(z)-\left(\Delta_{-\frac{1}{4}} f\right)^{2}=z-\frac{1}{16}$. However, $\delta(f) \neq \operatorname{deg} q(z)$ in the above two examples.

Corollary 1.3 Let $p(z) \not \equiv 0, q(z), r(z)$ be polynomials, $n>1$. Let $f(z)$ be finite order entire solutions of the equation

$$
f(z)^{n}+p(z) e^{q(z)} \Delta_{c} f=r(z)
$$

then $\delta(f)=\operatorname{deg} q(z)$.

According to (6), we will give a further discussion on the existence of meromorphic solutions. We get:

Theorem 1.4 Let $p(z) \not \equiv 0, q(z), r(z)$ be polynomials. Assume one of the following assertions holds:

(i) $n>1, f(z)$ is a finite order meromorphic function (not entire),

(ii) $n>1, r(z) \equiv 0$, and $f(z)$ is a finite order entire function with infinitely many zeros,

(iii) $n \geq 1, r(z) \not \equiv 0$, and $f(z)$ is a finite order entire function satisfying $\lambda(f)<\delta(f)$.

Then $f(z)$ cannot be a solution of (6).

Remarks (1) From Theorem 1.4, we know that the solution of the equation $f(z)^{n}+$ $p(z) e^{q(z)} \Delta_{c} f=0$ must be expressed as $f(z)=\alpha(z) e^{\beta(z)}$, where $\alpha(z)$ and $\beta(z)$ are polynomials.

(2) The condition that $n>1$ in Theorem 1.4 is sharp. In fact, if $n=1$, then we know the function $f(z)=\frac{e^{z}}{z}$ is a solution of the equation $f(z)+\left(\frac{z}{2 \pi i}+1\right) \Delta_{2 \pi i} f=0$. Moreover, the function $f(z)=\cos z$ can solve $f(z)+\frac{1}{2} \Delta_{\pi} f=0$.

(3) Some ideas of this paper are from [13].

\section{Preliminary lemmas}

Lemma 2.1 [14, Theorem 2.1] Let $f(z)$ be a meromorphic function of finite order, and let $c \in \mathbb{C}$, then

$$
m\left(r, \frac{f(z+c)}{f(z)}\right)+m\left(r, \frac{f(z)}{f(z+c)}\right)=S(r, f) .
$$


Remark From Lemma 2.1, we know $T(r, f)=T(r, f(z+c))+S(r, f)$ when $f(z)$ is an entire function of finite order.

Lemma 2.2 [15, Theorem 2.4.2] Let $f(z)$ be a transcendental meromorphic solution of

$$
f^{n} A(z, f)=B(z, f),
$$

where $A(z, f), B(z, f)$ are differential polynomials in $f$ and its derivatives with small meromorphic coefficients $a_{\lambda}$, in the sense of $m\left(r, a_{\lambda}\right)=S(r, f)$ for all $\lambda \in I$. If $d(B(z, f)) \leq n$, then $m(r, A(z, f))=S(r, f)$.

Lemma 2.3 [1, Theorem 1.56] Let $f_{j}(z)(j=1,2,3)$ be meromorphic functions that satisfy

$$
\sum_{j=1}^{3} f_{j}(z) \equiv 1
$$

If $f_{1}(z)$ is not a constant, and

$$
\sum_{j=1}^{3} N\left(r, \frac{1}{f_{j}}\right)+2 \sum_{j=1}^{3} \bar{N}\left(r, f_{j}\right)<(\lambda+o(1)) T(r),
$$

where $\lambda<1$ and $T(r)=\max _{1 \leq j \leq 3}\left\{T\left(r, f_{j}\right)\right\}$, then either $f_{2}(z) \equiv 1$ or $f_{3}(z) \equiv 1$.

Lemma 2.4 [1, Theorem 1.51] Suppose that $f_{j}(z)(j=1, \ldots, n)(n \geq 2)$ are meromorphic functions and $g_{j}(z)(j=1, \ldots, n)$ are entire functions satisfying the following conditions.

(1) $\sum_{j=1}^{n} f_{j}(z) e^{g_{j}(z)} \equiv 0$.

(2) $1 \leq j<k \leq n, g_{j}(z)-g_{k}(z)$ are not constants for $1 \leq j<k \leq n$.

(3) For $1 \leq j \leq n, 1 \leq h<k \leq n$,

$$
T\left(r, f_{j}\right)=o\left\{T\left(r, e^{g_{h}-g_{k}}\right)\right\}, \quad r \rightarrow \infty, r \notin E,
$$

where $E \subset(1, \infty)$ is of finite linear measure.

Then $f_{j}(z) \equiv 0$.

\section{Proof of Theorem 1.1}

If $q(z)$ is a constant or $r(z) \equiv 0$, then the conclusion follows from Theorem B. It remains to consider the case $q(z)$ is a non-constant polynomial and $r(z) \not \equiv 0$. Assume $f(z)$ is a transcendental entire solution of (4), which is finite order, not of period $c$. Differentiating (4) and eliminating $e^{q(z)}$, we have

$$
\begin{aligned}
& f(z)^{n-1}\left(n f^{\prime}(z)-\left(q^{\prime}(z)+\frac{r^{\prime}(z)}{r(z)}\right) f(z)\right) \\
& \quad=\left(q^{\prime}(z)+\frac{r^{\prime}(z)}{r(z)}\right) p(z)\left(\Delta_{c} f\right)^{m}-p^{\prime}(z)\left(\Delta_{c} f\right)^{m}-m p(z)\left(\Delta_{c} f\right)^{m-1}\left(\Delta_{c} f\right)^{\prime} .
\end{aligned}
$$

If $n f^{\prime}(z)-\left(q^{\prime}(z)+\frac{r^{\prime}(z)}{r(z)}\right) f(z) \equiv 0$, then we have $f(z)^{n}=A r(z) e^{q(z)}$. Writing $f(z)=h(z) e^{\frac{q(z)}{n}}$, where $h(z)$ satisfies $h(z)^{n}=\operatorname{Ar}(z)$ and $A$ is a non-zero constant. Substituting $f(z)$ into (4), 
we get

$$
(A-1) r(z) e^{q(z)}+p(z)\left(\Delta_{c} f\right)^{m} \equiv 0 .
$$

Clearly, if $A=1$, then $f(z)$ is a period function with period $c$, which contradicts the assumption. Hence, $A \neq 1$. Let $g=e^{\frac{q(z)}{n}}$, then $\left(\Delta_{c} f\right)^{m}$ can be expressed as $\sum_{i=0}^{m}\left(\begin{array}{c}m \\ i\end{array}\right)(-1)^{i} h(z)^{i} h(z+$ $c)^{m-i} g(z)^{i} g(z+c)^{m-i}$. Furthermore, from Lemma 2.1, we have

$$
\begin{aligned}
T\left(r,\left(\Delta_{c} f\right)^{m}\right) & =T\left(r, \sum_{i=0}^{m}\left(\begin{array}{c}
m \\
i
\end{array}\right)(-1)^{i} h(z)^{i} h(z+c)^{m-i} g(z)^{i} g(z+c)^{m-i}\right) \\
& =m\left(r, \sum_{i=0}^{m}\left(\begin{array}{c}
m \\
i
\end{array}\right)(-1)^{i} h(z)^{i} h(z+c)^{m-i} g(z)^{i} g(z+c)^{m-i}\right) \\
& \leq m\left(r, \frac{\sum_{i=0}^{m}\left(\begin{array}{c}
m \\
i
\end{array}\right)(-1)^{i} g(z+c)^{m-i} g(z)^{i}}{g(z)^{m}}\right)+m\left(r, g(z)^{m}\right)+S(r, g) \\
& \leq T\left(r, g(z)^{m}\right)+S(r, g)=m T(r, g)+S(r, g) .
\end{aligned}
$$

From (8) and (9), we get

$$
n T(r, g) \leq m T(r, g)+S(r, g)
$$

which contradicts the condition that $n>m$. Therefore, we conclude that $n f^{\prime}(z)-\left(q^{\prime}(z)+\right.$ $\left.\frac{r^{\prime}(z)}{r(z)}\right) f(z) \not \equiv 0$. We discuss the following two cases.

Case 1. $n>m+1$. Rewrite (7) in the following forms:

$$
\begin{aligned}
& f(z)^{n-m-1}\left(n f^{\prime}(z)-\left(q^{\prime}(z)+\frac{r^{\prime}(z)}{r(z)}\right) f(z)\right) \\
& =\left(q^{\prime}(z)+\frac{r^{\prime}(z)}{r(z)}\right) p(z) \frac{\left(\Delta_{c} f\right)^{m}}{f^{m}}-p^{\prime}(z) \frac{\left(\Delta_{c} f\right)^{m}}{f^{m}}-m p(z) \frac{\left(\Delta_{c} f\right)^{m-1}\left(\Delta_{c} f\right)^{\prime}}{f^{m}}
\end{aligned}
$$

and

$$
\begin{aligned}
& f(z)^{n-m-2}\left(f\left(n f^{\prime}(z)-\left(q^{\prime}(z)+\frac{r^{\prime}(z)}{r(z)}\right) f(z)\right)\right) \\
& =\left(q^{\prime}(z)+\frac{r^{\prime}(z)}{r(z)}\right) p(z) \frac{\left(\Delta_{c} f\right)^{m}}{f^{m}}-p^{\prime}(z) \frac{\left(\Delta_{c} f\right)^{m}}{f^{m}}-m p(z) \frac{\left(\Delta_{c} f\right)^{m-1}\left(\Delta_{c} f\right)^{\prime}}{f^{m}} .
\end{aligned}
$$

Applying Lemma 2.1, Lemma 2.2, and the lemma on the logarithmic derivative to the above two equations, we obtain

$$
T\left(r, n f^{\prime}-\left(q^{\prime}+\frac{r^{\prime}}{r}\right) f\right)=m\left(r, n f^{\prime}-\left(q^{\prime}+\frac{r^{\prime}}{r}\right) f\right)+S(r, f)=S(r, f)
$$

and

$$
T\left(r, f\left(n f^{\prime}-\left(q^{\prime}+\frac{r^{\prime}}{r}\right) f\right)\right)=m\left(r, f\left(n f^{\prime}-\left(q^{\prime}+\frac{r^{\prime}}{r}\right) f\right)\right)+S(r, f)=S(r, f)
$$


Hence

$$
T(r, f)=S(r, f)
$$

which is a contradiction.

Case 2. $n=m+1$. For this case, (4) and (7) now take the following forms:

$$
f^{m+1}+p(z)\left(\Delta_{c} f\right)^{m}=r(z) e^{q(z)}
$$

and

$$
\begin{aligned}
& f(z)^{m}\left((m+1) f^{\prime}(z)-\left(q^{\prime}(z)+\frac{r^{\prime}(z)}{r(z)}\right) f(z)\right) \\
& =\left(q^{\prime}(z)+\frac{r^{\prime}(z)}{r(z)}\right) p(z)\left(\Delta_{c} f\right)^{m}-p^{\prime}(z)\left(\Delta_{c} f\right)^{m}-m p(z)\left(\Delta_{c} f\right)^{m-1}\left(\Delta_{c} f\right)^{\prime} .
\end{aligned}
$$

Set $H(z)=(m+1) f^{\prime}(z)-\left(q^{\prime}(z)+\frac{r^{\prime}(z)}{r(z)}\right) f(z)$. Dividing (11) by $f(z)^{m}$, we have

$$
H(z)=\left(q^{\prime}(z)+\frac{r^{\prime}(z)}{r(z)}\right) p(z) \frac{\left(\Delta_{c} f\right)^{m}}{f^{m}}-p^{\prime}(z) \frac{\left(\Delta_{c} f\right)^{m}}{f^{m}}-m p(z) \frac{\left(\Delta_{c} f\right)^{m-1}\left(\Delta_{c} f\right)^{\prime}}{f^{m}} .
$$

Since $f(z)$ is entire, applying Lemma 2.1 and the lemma on the logarithmic derivative to the above equation, we conclude that

$$
T(r, H)=m(r, H)+S(r, f)=S(r, f) .
$$

Differentiating $H(z)$, we have

$$
\begin{aligned}
& (m+1) f^{\prime \prime}-\left(q^{\prime}+\frac{r^{\prime}}{r}\right) f^{\prime}-\left(q^{\prime}+\frac{r^{\prime}}{r}\right)^{\prime} f \\
& =\frac{H^{\prime}}{H} \cdot H=\frac{H^{\prime}}{H}\left((m+1) f^{\prime}-\left(q^{\prime}+\frac{r^{\prime}}{r}\right) f\right),
\end{aligned}
$$

that is

$$
(m+1) f^{\prime \prime}-\left(q^{\prime}+\frac{r^{\prime}}{r}+(m+1) \frac{H^{\prime}}{H}\right) f^{\prime}-\left(q^{\prime \prime}-q^{\prime} \frac{H^{\prime}}{H}+\left(\frac{r^{\prime}}{r}\right)^{\prime}-\frac{H^{\prime}}{H} \cdot \frac{r^{\prime}}{r}\right) f=0 .
$$

The above equation can be rewritten in the following form:

$$
\begin{aligned}
& (m+1)\left(\left(\frac{f^{\prime}}{f}\right)^{\prime}+\left(\frac{f^{\prime}}{f}\right)^{2}\right)-\left(q^{\prime}+\frac{r^{\prime}}{r}+(m+1) \frac{H^{\prime}}{H}\right) \frac{f^{\prime}}{f} \\
& -\left(q^{\prime \prime}-q^{\prime} \frac{H^{\prime}}{H}+\left(\frac{r^{\prime}}{r}\right)^{\prime}-\frac{H^{\prime}}{H} \cdot \frac{r^{\prime}}{r}\right)=0 .
\end{aligned}
$$

Suppose $z_{0}$ is a zero of $f(z)$ with multiplicity $k$. If $z_{0}$ is a zero of $r(z)$ as well, then the contribution to $N\left(r, \frac{1}{f}\right)$ is $S(r, f)$. Assuming that $z_{0}$ is not a zero of $r(z)$, we will discuss the two subcases: 
Subcase 1 . Suppose $z_{0}$ is a zero of $H(z)$ with multiplicity $t$. From (13), by simple calculations, we know that $k=1+t \leq 2 t$, which means that $z_{0}$ is a contribution of $S(r, f)$ to $N\left(r, \frac{1}{f}\right)$ by (12).

Subcase 2. Suppose $z_{0}$ is not a zero of $H(z)$. By (13), we get $k^{2}-k=0$, then such a zero of $f(z)$ must be simple and we find that $q^{\prime}+\frac{r^{\prime}}{r}+(m+1) \frac{H^{\prime}}{H}$ must vanish at $z_{0}$. That implies that $z_{0}$ makes a contribution of $S(r, f)$ to $N\left(r, \frac{1}{f}\right)$ by $(12)$.

In a word, $N\left(r, \frac{1}{f}\right)=S(r, f)$. Therefore, we can assume $f(z)$ to be of the form $f(z)=$ $G(z) e^{s(z)}$, where $s(z)$ is a non-constant polynomial, and $G(z)$ is an entire function satisfying $N\left(r, \frac{1}{G}\right)=T(r, G)=S(r, f)$ by the Hadamard factorization theorem. Substituting this expression into (10), we get

$$
G(z)^{m+1} e^{(m+1) s(z)}+p(z)\left(G(z+c) e^{s(z+c)}-G(z) e^{s(z)}\right)^{m}=r(z) e^{q(z)},
$$

and so

$$
\begin{aligned}
& G(z)^{m+1} e^{(m+1) s(z)}+p(z)\left(\sum_{i=0}^{m-1}\left(\begin{array}{c}
m \\
i
\end{array}\right)(-1)^{i} G(z+c)^{m-i} G(z)^{i} e^{(m-i) s(z+c)+i s(z)}\right) \\
& +p(z)(-1)^{m} G(z)^{m} e^{m s(z)}=r(z) e^{q(z)},
\end{aligned}
$$

which may be written in the form

$$
\begin{aligned}
& \frac{G(z) e^{s(z)}}{p(z)(-1)^{m-1}}+\frac{r(z) e^{q(z)}}{p(z)(-1)^{m} G(z)^{m} e^{m s(z)}} \\
& +\frac{\sum_{i=0}^{m-1}\left(\begin{array}{c}
m \\
i
\end{array}\right)(-1)^{i} G(z+c)^{m-i} G(z)^{i} e^{(m-i) s(z+c)+i s(z)}}{(-1)^{m-1} G(z)^{m} e^{m s(z)}}=1 .
\end{aligned}
$$

From Lemma 2.3, we have either

$$
f_{2}(z)=\frac{r(z) e^{q(z)}}{p(z)(-1)^{m} G(z)^{m} e^{m s(z)}} \equiv 1
$$

or

$$
f_{3}(z)=\frac{\sum_{i=0}^{m-1}\left(\begin{array}{c}
m \\
i
\end{array}\right)(-1)^{i} G(z+c)^{m-i} G(z)^{i} e^{(m-i) s(z+c)+i s(z)}}{(-1)^{m-1} G(z)^{m} e^{m s}(z)} \equiv 1
$$

If $f_{2}(z) \equiv 1$, then we get

$$
r(z) e^{q(z)}=p(z)(-1)^{m} G(z)^{m} e^{m s(z)}=p(z)(-1)^{m} f(z)^{m} .
$$

Plugging the above equation into (10), similar to (9), we get $T\left(r, r e^{q}-p\left(\Delta_{c} f\right)^{m}\right) \leq m T(r, f)+$ $S(r, f)$. Comparing both sides of (9), we get a contradiction. If $f_{3}(z) \equiv 1$, then we get $f(z)^{m+1} \equiv r(z) e^{q(z)}$, which means that $\Delta_{c} f \equiv 0$, and this contradicts the assumption.

Therefore, from the discussions above, we find that a transcendental finite order entire function $f(z)$ which is not of period $c$, cannot be a solution of (4). The proof of Theorem 1.1 is finished completely. 


\section{Proof of Theorem 1.2}

Applying Lemma 2.1 to (5), we get

$$
\begin{aligned}
n m(r, f) & =m\left(r, r-p e^{q}\left(\Delta_{c} f\right)^{m}\right) \\
& \leq m\left(r, e^{q}\right)+m\left(r, \frac{\left(\Delta_{c} f\right)^{m}}{f^{m}}\right)+m\left(r, f^{m}\right)+S(r, f) \\
& \leq m\left(r, e^{q}\right)+m m(r, f)+S(r, f),
\end{aligned}
$$

which means that $(n-m) m(r, f) \leq m\left(r, e^{q}\right)+S(r, f)$. From the assumption that $n>m$ and the above inequality, we conclude that $\delta(f) \leq \operatorname{deg} q(z)$.

Now we show that $\delta(f)=\operatorname{deg} q(z)$. Suppose to the contrary that $\delta(f)<\operatorname{deg} q(z)$. Then $\delta\left(f^{n}+p e^{q}\left(\Delta_{c} f\right)^{m}\right)=\operatorname{deg} q(z)>0$ from Remark below Lemma 2.1, and $\delta(r)=0$. This is a contradiction by (5).

\section{Proof of Theorem 1.4}

First, we confirm that one period function with period $c$ cannot be a solution of (6). Actually, if $f(z)$ is a period function, then the order of $f(z)$ satisfies $\delta(f) \geq 1$. At the same time, we get $f(z)^{n}=r(z)$, which is impossible. It remains to consider the function which is not of period $c$.

(i) Suppose that $f(z)$ is a finite order meromorphic solution of (6), and $z_{0}$ is a pole of $f(z)$ with multiplicity $t$. Then we get $z_{0}+c$ is a pole of $f(z)$ with multiplicity $\geq n t$. By calculation, we see that $z_{0}+k c$ is a pole of $f(z)$ with multiplicity $n^{k} t$, where $k$ is a positive integer. Since $n>1$, we conclude that $\lambda(f)=\infty$, i.e., $\delta(f)=\infty$. This is a contradiction.

(ii) Suppose that a finite order entire solution $f(z)$ has infinitely many zeros and $r(z) \equiv 0$. We assume that all zeros of $p(z)$ are in $D=\{z:|\operatorname{Re} z| \leq M,|\operatorname{Im} z| \leq M\}$, where $M>0$ is some constant. From the assumption, we know that $f(z)$ has infinitely many zeros which are not in $D$. If $z_{0} \notin D$ satisfies $f\left(z_{0}\right)=0$, then from (6) we know that $z_{0}+k c$ are zeros of $f(z)$. Moreover, we have $z_{0}+k c$ are not in $D$, as $k \rightarrow \infty$. In the same way as (i), we get $\lambda\left(\frac{1}{f}\right)=\infty$, i.e., $\delta(f)=\infty$, which contradicts the assumption that $\delta(f)<\infty$.

(iii) Suppose $f(z)$ is an entire solution of finite order satisfying $\lambda(f)<\delta(f)$, then we know $\delta(f) \geq 1$ by the conclusion of Corollary 1.3. Hence, from the Hadamard factorization theorem, $f(z)$ can be written as $f(z)=\alpha(z) e^{\beta(z)}$, where $\beta(z)$ is a non-constant polynomial.

Case 1. $n>1$. From the conclusion of Corollary 1.3, we know $\lambda(f)=\delta(\alpha)<\delta(f)=$ $\operatorname{deg} q(z)=q \geq 1$. So we can rewrite $f(z)$ in the following form:

$$
f(z)=\gamma(z) e^{a_{q} z^{q}}
$$

where $a_{q}$ is a non-zero constant, $\delta(\gamma)<q$, and $T(r, \gamma)=S(r, f)$. From the conclusion of Corollary 1.3, we conclude that

$$
e^{q(z)}=\mu(z) e^{b_{q} z^{q}},
$$


where $b_{q}$ is a non-zero constant, $\delta(\mu)<q$, and $T(r, \mu)=S(r, f)$. By (6), (15), and (16), we have

$$
\gamma(z)^{n} e^{n a_{q} z^{q}}+p(z) \mu(z)\left(\gamma(z+c) e^{\nu(z)}-\gamma(z)\right) e^{\left(a_{q}+b_{q}\right) z^{q}}=r(z),
$$

where $v(z)$ is a polynomial such that $\operatorname{deg} v(z) \leq q-1$.

Subcase 1. If $n a_{q}=b_{q}+a_{q}$, then (17) can be expressed as

$$
\gamma(z)^{n}+p(z) \mu(z)\left(\gamma(z+c) e^{\nu(z)}-\gamma(z)\right)=\frac{r(z)}{e^{n a_{q} z^{q}}}
$$

Since $r(z) \not \equiv 0$, we get $\gamma(z)^{n}+p(z) \mu(z)\left(\gamma(z+c) e^{\nu(z)}-\gamma(z)\right) \not \equiv 0$. Comparing the characteristics function of both sides of the above equation, we get a contradiction.

Subcase 2. If $n a_{q} \neq b_{q}+a_{q}$, then we rewrite (17) in the form

$$
\gamma(z)^{n} e^{n a_{q} z^{q}}+p(z) \mu(z)\left(\gamma(z+c) e^{\nu(z)}-\gamma(z)\right) e^{\left(a_{q}+b_{q}\right) z^{q}}-r(z)=0 .
$$

Applying Lemma 2.4 to the above equation, we get $r(z) \equiv 0$, which contradicts the assumption.

Case 2. $n=1$. Comparing both sides of (6), we obtain $\delta(f) \geq \operatorname{deg} q(z)=q$ by Remark below Lemma 2.1. If $\delta(f)=q$, then in the same way as Case 1, the conclusion follows. If $k=\delta(f)>q$, then $f(z)$ can be written as $f(z)=\phi(z) e^{a_{k} z^{k}}$, where $\delta(\phi)<k$, and $T(r, \phi)=S(r, f)$. Substituting this expression into (6), we get

$$
\left(\phi(z)+p(z) e^{q(z)}(\phi(z+c) \varphi(z)-\phi(z))\right) e^{a_{k} z^{k}}=r(z),
$$

where $\delta(\varphi)<k$, and $T(r, \varphi)=S(r, f)$. Since $r(z) \not \equiv 0$, we get $\phi(z)+p(z) e^{q(z)}(\phi(z+c) \varphi(z)-$ $\phi(z)) \not \equiv 0$. Comparing the characteristics function of both sides of the above equation, we get a contradiction.

\section{Competing interests}

The authors declare that they have no competing interests.

Authors' contributions

XQ completed the main part of this paper, JD and LY corrected the main theorems. All authors read and approved the final manuscript.

\section{Author details}

${ }^{1}$ School of Mathematics, University of Jinan, Jinan, Shandong 250022, P.R. China. ${ }^{2}$ Quancheng Middle School, Jinan, Shandong 250000, P.R. China. ${ }^{3}$ School of Mathematics, Shandong University, Jinan, Shandong 250100, P.R. China.

\section{Acknowledgements}

The authors thank the referee for his/her valuable suggestions to improve the present paper. This work was supported by the National Natural Science Foundation of China (No. 11301220 and No. 11371225) and the Tianyuan Fund for Mathematics (No. 11226094), the NSF of Shandong Province, China (No. ZR2012AQ020) and the Fund of Doctoral Program Research of University of Jinan (XBS1211).

\section{Received: 23 April 2014 Accepted: 17 September 2014 Published: 03 Oct 2014}

\section{References}

1. Yang, CC, Yi, HX: Uniqueness Theory of Meromorphic Functions. Kluwer Academic, Dordrecht (2003)

2. Yang, CC: On entire solutions of a certain type of nonlinear differential equation. Bull. Aust. Math. Soc. 64, 377-380 (2001)

3. Heittokangas, J, Korhonen, R, Laine, I: On meromorphic solutions of certain nonlinear differential equations. Bull. Aust. Math. Soc. 66, 331-343 (2002) 
4. Li, P, Yang, CC: On the nonexistence of entire solution of certain type of nonlinear differential equations. J. Math. Anal. Appl. 320, 827-835 (2006)

5. Li, P: Entire solution of certain type of differential equations. J. Math. Anal. Appl. 344, 253-259 (2008)

6. Yang, CC, Laine, I: On analogies between nonlinear difference and differential equations. Proc. Jpn. Acad., Ser. A, Math. Sci. 86, 10-14 (2010)

7. Liu, K, Liu, XL, Yang, LZ: Existence of entire solutions of nonlinear difference equations. Czechoslov. Math. J. 61 , 565-576 (2011)

8. Liu, K, Cao, TB: Entire solutions of Fermat type q-difference differential equations. Electron. J. Differ. Equ. 2013, 59 (2013)

9. Peng, CW, Chen, ZX: On a conjecture concerning some nonlinear difference equations. Bull. Malays. Math. Sci. Soc. 36, 221-227 (2013)

10. Wen, ZT, Heittokangas, J, Laine, I: Exponential polynomials as solutions of certain nonlinear difference equations. Acta Math. Sin. 28, 1295-1306 (2012)

11. Qi, XG: Value distribution and uniqueness of difference polynomials and entire solutions of difference equations. Ann. Pol. Math. 102, 129-142 (2011)

12. Gross, F: On the equation $f^{n}+g^{n}=1$. Bull. Am. Math. Soc. $72,86-88$ (1966)

13. Laine, I: A note on value distribution of difference polynomials. Bull. Aust. Math. Soc. 81, 353-360 (2010)

14. Halburd, R, Korhonen, R: Nevanlinna theory for the difference operator. Ann. Acad. Sci. Fenn., Math. 31, 463-478 (2006)

15. Laine, I: Nevanlinna Theory and Complex Differential Equations. de Gruyter, Berlin (1993)

10.1186/1687-1847-2014-256

Cite this article as: Qi et al.: The properties of solutions of certain type of difference equations. Advances in Difference Equations 2014, 2014:256

\section{Submit your manuscript to a SpringerOpen ${ }^{\ominus}$ journal and benefit from:}

- Convenient online submission

- Rigorous peer review

- Immediate publication on acceptance

Open access: articles freely available online

- High visibility within the field

- Retaining the copyright to your article 